\title{
Intrinsic and extrinsic influences on self-recognition of actions
}

\author{
Akila Kadambi ${ }^{1}$ \\ Qi Xie ${ }^{1}$ \\ Hongjing $\mathrm{Lu}^{1,2}$ \\ ${ }^{1}$ Department of Psychology, University of California, Los Angeles, 502 Portola Plaza, Los Angeles, CA, \\ United States, 90095 \\ ${ }^{2}$ Department of Statistics, University of California, Los Angeles, 520 Portola Plaza, Los Angeles, CA, \\ United States, 90095
}

\begin{abstract}
Despite minimal visual experience and unfamiliar third-person viewpoints, humans are able to recognize their own body movements even when actions are reduced to point-light displays. What factors influence visual self-recognition of own actions? To address this question, we recorded whole-body movements of a large sample of participants $(N=101)$ performing a range of actions. After a delay period, participants were tested in a self-recognition task: identifying own actions depicted in point-light displays amongst three other point-light actors performing identical actions. While participants showed above-chance accuracy on average for self-recognition, we found substantial differences in performance across actions and individuals. Self-recognition performance was modulated by interactions between extrinsic factors (associated with the degree of motor planning in performed actions) and intrinsic traits linked to individuals' motor imagery ability and sensorimotor self-processing ability (autism and schizotypal traits). These interactions shed light on mechanistic possibilities for how the motor system may augment vision to construct the core of self-awareness.
\end{abstract}

Keywords: self-recognition, body movement, action, individual differences, self-awareness

Corresponding author: akadambi@ucla.edu

\section{Public Significance Statement:}

In this large-scale study on self-action recognition, we demonstrate how motor mechanisms influence self-recognition of own actions beyond visual features. Our individual difference approach further introduces novel interactions between intrinsic participant and extrinsic motor factors during self-action recognition. 


\section{Running head: SELF-RECOGNITION OF ACTIONS}

\section{Introduction}

Recognition of oneself depends on more than visual experience. As a prime example, humans can recognize their own actions from minimal visual input provided by point-light action displays (Cutting \& Kozlowski, 1977; Loula et al., 2005; Beardsworth \& Buckner, 1981)— disconnected dot animations depicting the motion of key joints on the human body (Johansson, 1973). Despite removal of body identity information and the depiction of actions from an unfamiliar third-person perspective, selfrecognition from point-light movements suggests that people can readily connect motor experience of performing actions to visual recognition of actions. This finding is consistent with multiple lines of evidence that have revealed close links between motor and visual representations of actions (e.g., Binet, 1886; Viviani \& Stucchi, 1992; Prinz, 1997; Knoblich \& Flach, 2001; Casile \& Giese, 2006; Rizzolatti, Fogassi, \& Gallese, 2001; Blake \& Shiffrar, 2007).

Previous research has identified a few core characteristics of self-recognition from actions. From point-light actions, participants are more accurate in recognizing themselves than visually familiar friends (Cutting \& Kozlowski, 1977), even when configural topographic cues are disrupted (Cook, Johnston, \& Heyes, 2012; cf. Loula et al., 2005), or visual feedback is removed (Casile \& Giese, 2006). People can identify their own actions from hand movements (Conson, Aromino \& Trojano, 2010) and from whole-body movements (Loula et al., 2005). Self-recognition of one's own gait is viewpointinvariant (cf. Mitchell \& Curry, 2016), but recognition of familiar friends from their gait patterns is viewpoint-dependent, with better performance from frontal than profile views (Jokisch, Daum \& Troje, 2006). View-independent performance for self-recognition from point-lights has also been observed for actions less common than walking (Loula et al., 2005; Burling et al., 2019), suggesting that visual representation of self-actions based primarily on motor experience is fundamentally different from the action representation of others learned from visual experience. Furthermore, the self-action advantage extends beyond explicit visual recognition, influencing the predictive accuracy of future action 


\section{Running head: SELF-RECOGNITION OF ACTIONS}

outcomes (e.g., Knoblich \& Flach, 2001; Bischoff et al., 2012; cf. Hohmann et al., 2011), taskirrelevant, body part recognition (Frassinetti et al., 2009; Daprati \& Sirigu, 2002; Daprati, Weriessneggeer, \& Lacquaniti, 2007), facial expression recognition (Cook, Johnson \& Heyes, 2012), memory for self-performed action verbs (i.e., enactment) (Engelkamp \& Krumnacker, 1980), and multimodal action recognition (e.g., Repp \& Knoblich, 2004; Flach, Knoblich, \& Prinz, 2004; Murgia et al., 2012; Kennel et al., 2014).

While these studies reveal the importance of the motor experience for self-action recognition, further research is needed to pin down exactly how the motor system contributes to self-action recognition. Several limitations of previous studies must be addressed. First, studies of self-recognition from point-light movements have all utilized relatively small sample sizes of participants (in the range of 6 34). Second, there lacks systematic assessments as to how different actions influence selfrecognition performance across different actions, although some previous studies demonstrated the presence of such item-level influences (Loula et al., 2005; Sevdalis \& Keller, 2009; Burling et al., 2019). Loula et al. (2005) included ten actions in their study and found that self-recognition performance varied across individual actions, with more accurate self-recognition for visually complex actions such as dancing or boxing, than walking/running. Sevdalis and Keller (2009) found greater self-recognition performance for improvised dancing compared to stereotyped gait patterns. Coste et al (2020) asked participants to perform postural motions with general instructions, such as "create postural motions by keeping knees extended with toes and heels in constant contact with the floor." The study found that people more often misidentified themselves for movements that showed high similarity between self and other actions. However, these studies have yet to systematically manipulate and compare actions with different levels of perceptual variability, that are more specifically modulated by the degree of motor planning required by the action goal (e.g., Molnar-Szakacs et al., 2006; Li et al., 2020). Third, beyond item-level variability in action stimuli, another limitation in the literature on self-action recognition 


\section{Running head: SELF-RECOGNITION OF ACTIONS}

49 relates to the impact of intrinsic traits at the participant level—opting for group-level rather than

50 individual differences. To date, only one behavioral study from our group (Burling et al., 2019) has

51 examined the impact of autistic traits on self-action recognition performance. However, the study

52 focused on group comparisons by selecting participants with extremely high or low autistic traits, while

53 neither mapping out any other individual difference measures nor including a large sample of

54 participants to examine the variability of individual differences in self-action recognition.

To address the prior limitations, we conducted the first large-scale study $(N=101)$ in self-action

recognition to examine how motor-based mechanisms, linked to extrinsic factors of action stimuli,

interact with intrinsic traits of participants to impact self-recognition of actions. To assess extrinsic

influences that may impact item-level variability in self-recognition, the present study included a larger

range of actions, varying complexity based on the degree of motor planning involved in performing

actions. To examine intrinsic influence of participant-level variability, we assessed the relationship

61 between self-recognition performance and three individual difference measures: autistic, schizotypal, and motor imagery traits.

Numerous studies have shown atypical self-processing in two prevalent clinical conditions:

64 Autism Spectrum Conditions (ASC) - from the Greek root "autos” for self (Kanner 1943; Asperger, 1944; Perrykkad \& Howhy, 2020), and Schizophrenia Spectrum Conditions (SSC), a "disordered self”, characterized by “a disunity of consciousness” (Bleuler, 1911; Schneider 1959; Sass \& Parnas, 2003;

67 Kraeplin 1919; Parnas \& Herniksen, 2014; Sandsten et al., 2020). Recent research has attributed these types of atypicalities to a specific alteration in sensorimotor self-processing in clinical populations (e.g.,

69 Noel et al., 2016; Sass \& Borda, 2015; Gallese \& Ferri, 2014; Gessaroli et al., 2013; Perrykkad and

70 Howe, 2020). Researchers have suggested that own-body discrimination manifests in contrasting ways

71 in ASC and SSC (Noel, Cascio, Wallace, \& Park, 2016), and appears to relate to sharpened or blurred

72 internal self/other boundaries, respectively (Noel et al., 2016; Fletcher \& Frith, 2009). Consistent with 


\section{Running head: SELF-RECOGNITION OF ACTIONS}

73 sensorimotor characterizations of self-processing in ASC and SSC, converging studies in biological motion perception also reveal atypical performance patterns in action recognition related to both clinical disorders (e.g., Hur et al., 2016; Wang et al., 2018; Blain, Peterman, \& Park, 2017; Ferroni et al., 2020; Thakkar et al., 2011).

Many findings of perceptual atypicalities in clinical populations have been extended to individual differences in the broader spectrum of autistic traits and schizotypal traits within the general population (Miller \& Saygin, 2013; Puglia \& Morris, 2017; Thurman et al., 2016; van Boxtel et al., 2017; Wang et. al., 2018; Chakraborty \& Chakrabarti, 2015). The prototypical measurement of selfreported autism-spectrum traits in the general population is the Autism-Spectrum Quotient (AQ) questionnaire (Baron-Cohen et al., 2001). Recent evidence has identified an overlapping genetic and biological etiology underlying ASC and autistic traits (Bralten et al., 2017), in addition to behavioral overlap (Baron-Cohen et al., 2001). Several studies have reported a relation between AQ scores and performance in a range of tasks related to biological motion perception (e.g., Miller \& Saygin, 2013; Ahmed \& Vander Wyk, 2013; van Boxtel, Peng, Su, \& Lu, 2017, Blain, Peterman, \& Park, 2017). Similarly, researchers have developed individual measures of schizotypy traits assessed by the Schizotypal Personality Questionnaire (SPQ) (Raine, 1991), which estimates vulnerability toward developing schizophrenia, i.e., "psychosis-proneness" (Johns \& Van Os, 2001; Vollema et al., 2002). Schizotypal traits capture stable forms of the pathology (Widiger, 2012) and the spectrum of SSC in the neurotypical population (Torbet, Schulze, Fiedler, \& Reuter, 2015; Siever \& Davis, 2004). Previous research has also reported a relation between SPQ scores and performance on action recognition tasks 93 (e.g., Blain, Peterman, \& Park, 2017; Kim et al., 2011). In addition to autistic and schizotypal traits, we included motor imagery ability as a third intrinsic

95 factor. Motor imagery serves as a part of a simulation network, triggered either through visual observation or through self-imaging, since action recognition and simulation share overlapping neural and behavioral 


\section{Running head: SELF-RECOGNITION OF ACTIONS}

97 resources (Jeannerod \& Pacherie, 2004). We assessed motor imagery ability using the Vividness of Motor

98 Imagery Questionnaire-2 (Roberts, 2008) as a potential source of individual variability in self-recognition

99 of actions. This factor was examined for several reasons. First, recognition of one's own action requires

100 an egocentric (first-person) to allocentric (third-person) spatial simulation of one's own body (e.g.,

101 Ganesh, Schie, Cross, Lange, \& Wigboldus, 2015), and self-processing recruits motor imagery

102 mechanisms (e.g., Conson et al., 2010). Second, action perception and motor imagery representations

103 share common neural resources (Miller \& Saygin, 2013; Iacoboni \& Dapretto, 2006; Clark, Tremblay, \&

104 Ste-Marie, 2004; Grèzes \& Decety 2001), impacting variability in the general population on biological

105 motion tasks (Miller \& Saygin, 2013). In addition, both SSC and ASC have been linked to atypical motor

106 simulation ability (e.g., Oberman \& Ramchandran, 2007), with likely extensions to the general population.

107 The experimental design consisted of two sessions. In the first session, 27 actions performed by

108 each participant were recorded through motion capture. Participants were informed that their actions

109 were being recorded for an action recognition study, but were never informed of study's focus on self-

110 recognition. Eighteen actions were verbally instructed (i.e., "please naturalistically perform the action: to

111 grab"), and the other nine actions were elicited by video instruction, asking participants to imitate body

112 movements of an actor shown in a video. We included video instruction since imitation presents a

113 unique action requirement: integrating observed visual information with one's internal motor system

114 (i.e., observation of others performing actions and action production with self-body movements).

115 Additionally, action imitation involves lessened motor planning, due to copying another's motor

116 sequence (see enactment effect; Zimmer \& Engelkamp, 1996). If self-recognition performance depends

117 primarily on the degree of motoric planning involved in performing actions, we would expect that

118 imitation actions may yield lower accuracy in identifying own body movements than actions with verbal

119 labels. Using videos to prompt participants to imitate actions further removes the potential influence of

120 individual differences in language comprehension or in interpretation of semantic labels of actions. 


\section{Running head: SELF-RECOGNITION OF ACTIONS}

After a delay of about one month on average, participants returned to complete a self-recognition task that required identifying their own action among three other actors performing the same action. We hypothesized that participants would be able to self-recognize most actions above chance, and selfrecognition performance would be influenced by both extrinsic factors - action types linked to motor planning (Loula et al., 2005; Burling et al., 2019) - and intrinsic factors, linked to sensorimotor selfprocessing based on individual differences in autistic traits, schizotypal traits and motor imagery ability. To further separate the impact of motor experience from visual experience in self-action recognition (speculated upon in Loula et al., 2005), we included an additional visual recognition task in which participants were asked to recognize previously-seen actions in the imitation condition.

Method

Participants 108 undergraduate students $\left(M_{\mathrm{age}}=21.20, S D_{\mathrm{age}}=3.81\right.$, females $=79$, males $\left.=29\right)$ were recruited through the Subject Pool at the University of California, Los Angeles. Sample size was determined in accord with a previous study on the relation between emotion discrimination from the gait patterns and autistic and schizotypy traits (e.g., Blain, Peterman, and Park, 2017). The study was approved by the UCLA Institutional Review board. All participants were provided course credit for their participation and were naïve to the purpose of the study. Participants had normal or corrected-to-normal vision and no physical disabilities. Seven participants were excluded due to inputting errors of participant motion capture files, resulting in a total of 101 participants included in the analysis $(\mathrm{M}=28, \mathrm{~F}=73)$.

Apparatus Participants' body movements were recorded using the Microsoft Kinect V2.0 and Kinect SDK in a quiet testing room. Here, participants were instructed to perform the actions in a rectangular $0.76 \mathrm{~m} \times 1.52 \mathrm{~m}$ space, in order to provide flexibility to perform the action, while remaining within recording distance. The Kinect was placed $1.52 \mathrm{~m}$ above the floor and $2.59 \mathrm{~m}$ away from the participant. 


\section{Running head: SELF-RECOGNITION OF ACTIONS}

145 The three-dimensional (X-Y-Z) coordinates of the key joints were extracted at a rate of approximately 33

146 frames per second and later used to generate point-light displays of actions (see Figure 1). Customized

147 software developed in our lab was utilized to enhance movement signals, and to carry out additional

148 processing and trimming for actions presented later in the testing phase (van Boxtel \& Lu, 2013).

150 Stimuli Generation. All actions were selected to be commonly encountered actions and were captured

151 by the motion capture system. The actions varied in complexity in order to characterize a broad range of

152 common movements in daily life. First, participants performed nine simple and nine complex actions

153 provided with clear verbal labels (i.e., verbal instruction). Simple and complex actions were extended

154 from Burling et al (2019), delineated by whether the action was a simple goal (e.g., wave), conveying a 155 routine action with less motor planning, or a complex goal (e.g., argue) linked to more detailed motor 156 planning. The simple actions included grab, jump, wave, lift, kick, hammer, push, point, punch. The 157 complex actions included: argue, macarena, wash windows, play baseball, get attention, hurry up, fight, 158 stretch, and play guitar.

In addition, participants were performed actions based on video instruction. Participants were 160 asked to view an action performed by actors shown in a stick-figure display (i.e., lines connected between 161 joint positions) on a computer, and subsequently imitate the seen action afterwards. Nine imitation actions 162 were selected from the Carnegie Mellon Graphics Lab Motion Capture Database available online 163 ( $\underline{\text { http://mocap.cs.cmu.edu }), ~ g e n e r a t e d ~ f r o m ~ p r e-d e f i n e d ~ a c t o r s . ~ T h e ~ a c t i o n s ~ w e r e ~ s e l e c t e d ~ t o ~ c a p t u r e ~ a ~}$ 164 broad range of goal variability: jumping jacks, basketball, bend, direct traffic 1, direct traffic 2, 165 conversation, laugh, digging a hole, and chopping wood. To account for any implicit goal-attribution or 166 familiarity with action sequences that could impact the degree and/or type of imitation (e.g., Bekkering et 167 al., 2000; Wöhlschalger et al., 2003; Wild et al., 2009), we also included a range of familiar (e.g., bend) 168 and unfamiliar (e.g., directing traffic) imitation action sequences. Participants were never provided the 


\section{Running head: SELF-RECOGNITION OF ACTIONS}

169 verbal label for imitation actions. Each video displayed an actor shown as a stick figure performing one

170 of the imitation actions and was presented in three different angles to the subject, either to the right or left

$171\left(+/-45^{\circ}\right.$; half-profile $)$ or facing forward $\left(0^{\circ}\right.$; frontal $)$ by rotating the horizontal axis. Each imitation action

172 was recorded twice: once after viewing the three different angles, and once more after viewing only the

173 forward-facing angle. The first imitation recording was discarded (served as practice during motion

174 recording), and only the second imitation recording was used in the self-recognition experiment. The

175 recorded raw motion data from the Kinect system were passed through a double exponential adaptive

176 smoothing filter (LaViola, 2003) to remove noisy and jittered movements (e.g., ballistic random jump of

177 points). Additionally, the stimuli were trimmed and processed to display the point light-displays using

178 BioMotion Toolbox (van Boxtel \& Lu, 2013) with their segmented action recording, which would be 179 reiteratively looped in the self-recognition session.

\section{Procedure}

The experiment was split into two phases: motion recording and recognition. The first phase consisted of a motion recording session, where participants performed the actions and were recorded with

184 a motion capture system. Participants were informed that their actions were recorded for an action 185 recognition study and were never informed about the aim of the study. After a delay period $(M=37.39$ 186 days, $S D=5.20$ days; range: $23-56$ days), participants returned for the second phase to complete two 187 recognition tasks. In the first task, participants completed a self-recognition task by identifying their own 188 actions that were recorded with the motion capture system. In the second task, participants completed a 189 visual recognition task, where they identified the actions imitated during the motion recording session 190 (further elaboration below). The order of the self-recognition task and the visual recognition task was 191 counterbalanced between participants.

\section{Motion Recording Phase}




\section{Running head: SELF-RECOGNITION OF ACTIONS}
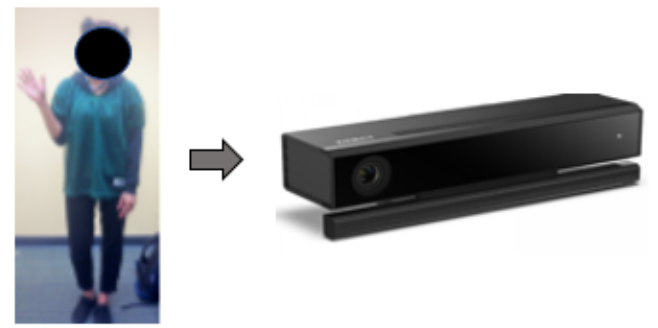

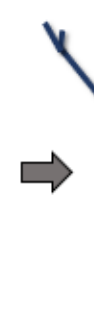

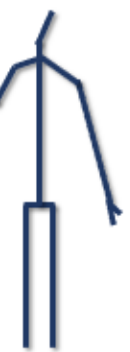
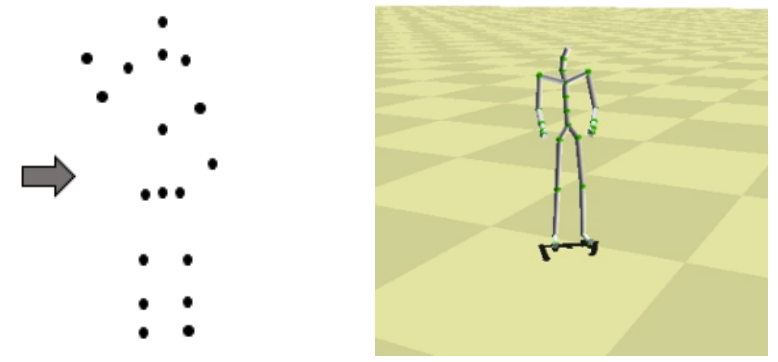

Figure 1. Illustration of motion recording. Left, actions were recorded by the Kinect system and converted to point-light displays; Right, an example frame of imitation action videos by an actor shown in a stickfigure display. Participants were asked to imitate the seen actions.

After completing the action recording, participants completed two questionnaires: Schizotypal Personality Questionnaire (SPQ; Raine, 1991) and the revised Vividness of Motor Imagery Questionnaire (VMIQ-2; Roberts et al., 2008). The SPQ was administered to assess degrees of schizotypal traits among individuals in the typical population. The VMIQ-2 was included to assess motor imagery differences as a potential source of variability in self-processing and biological motion processing.

\section{Recognition Phase: Self-Recognition Task and Visual-Recognition Task}

In the second phase (recognition test), participants were seated approximately 0.76 meters in front of a monitor in a dimly lit room and were asked to select their own action amongst three other distractor actions spread out horizontally along the center of the screen, as shown in Figure 2. Each action was presented with 25 point-lights located at key joints, in three different orientations (rotated around the vertical axis $0^{\circ}$, (frontal), $45^{\circ}$ (half-profile, right), $225^{\circ}$ (half-profile, left), for a total of 81 trials. All of the actions within a trial displayed the same orientation. The actions were looped until the participant 
215 selected one of the four horizontally-spread, randomly arranged boxes, or until a time period of 30

216 seconds. Participants were not provided any feedback. Participants were instructed to select their own

217 point-light action amongst four displays. The four animations included their own action and the same

218 action performed by three distractor actors with the same gender, all of whom participants were unfamiliar

219 with. All the point-light actions were normalized for maximum height. Following selection of the action, 220 participants were asked to provide a confidence judgment, in which they rated the confidence of their 221 selection from 1 (not at all confident) to 5 (most confident).

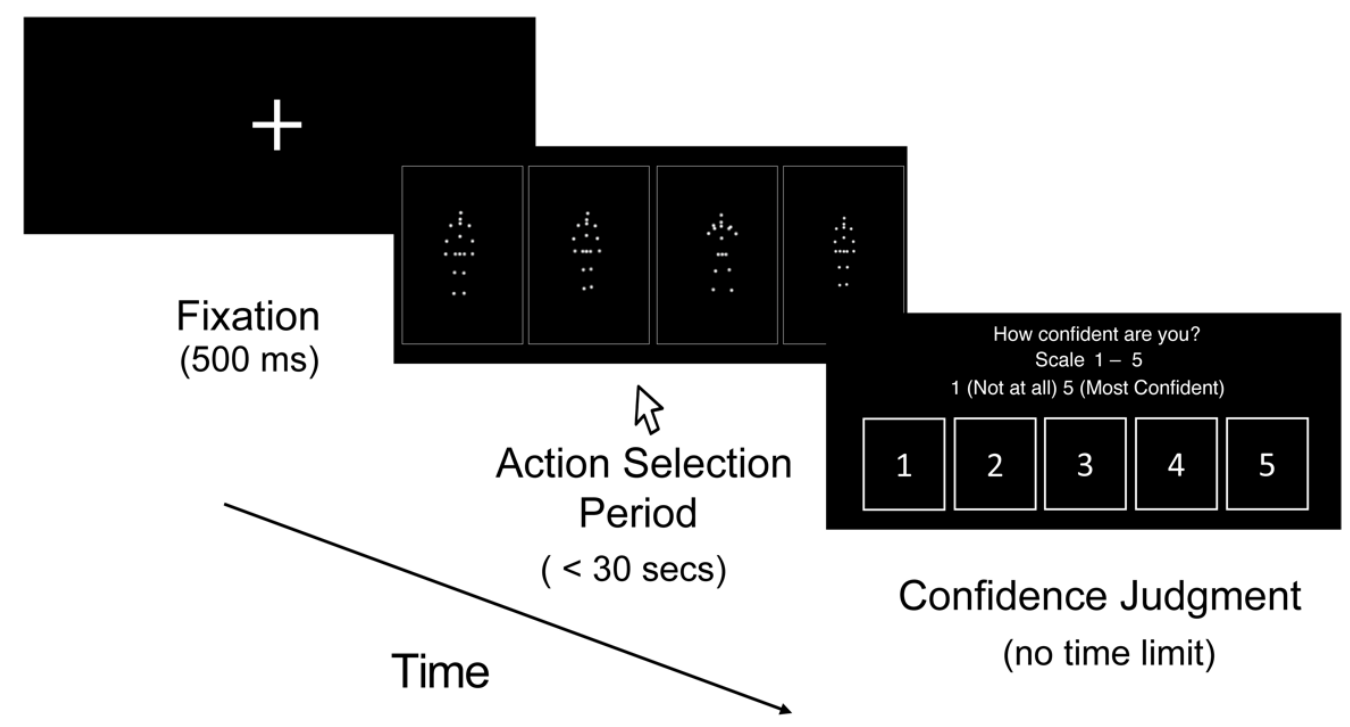

Figure 2. Schematic illustration of a sample trial showing wave action for the self-recognition task. One point-light action is the participant's action, while the other three point-light actions are distractors performed by gender-matched actors. During the display of actions, participants were provided a maximum of 30 seconds to click on the action that was their own. Following the actions, participants rated their overall confidence in the selection (1: not at all confident to 5: most confident).

230 participants were tested with both self-recognition task and an additional visual recognition task consisting

231 of nine trials displaying only the forward-facing imitation actions. Participants were instructed to identify

232 the stick-figure actor they imitated during the motion recording phase (converted to point-light display)

233 amongst three other distractor point-light actors who performed the same action, that were identical to 


\section{Running head: SELF-RECOGNITION OF ACTIONS}

234 those used in the self-recognition task. The inclusion of this visual recognition task allowed us to assess 235 whether recognition performance would differ when delineating self-generated from visually observed 236 actions. Importantly, while the stimulus layout of the task was identical to the self-recognition task, the 237 participants' own action from the self-recognition task was replaced by the original imitation actor from 238 the motion capture database, consequently introducing prior visual experience. As a result, participants 239 never viewed their own self-generated action in the visual recognition task. The order of presentation of 240 the visual recognition task was counterbalanced to either follow or precede the self-recognition task. 241 Following the self-recognition and visual recognition task, participants were asked to complete the 242 Autism-Spectrum Quotient (AQ) questionnaire to assess their degree of Autistic traits (Baron-Cohen et 243 al., 2001).

\section{Individual Difference Measures}

246 Autistic Quotient ASC resides on a spectrum of psychopathology. We assessed self-reported autism247 spectrum traits in the general population using the Autism-Spectrum Quotient (AQ) questionnaire, 248 consisting of 50 questions designed to measure five different subtypes: social skill, attention switching, 249 attention to detail, social communication, and imagination (Baron-Cohen et al., 2001). Response criteria 250 requires the selection of one of the four possibilities (four-point scale): "definitely disagree", "slightly 251 disagree", "slightly agree", "definitely agree." While not a diagnostic instrument, scores of $32+$ on the AQ

252 in the general population are generally indicative of a predisposition to ASC, out of a maximum score of 25350 points ( 1 point per question validating autism-spectrum traits), while the cutoff for ASC individuals on 254 the AQ is typically greater than 26 (Woodbury-Smith, Robinson, Wheelwright, \& Bardon-Cohen, 2005; 255 Ruzich et al., 2016).

256 Schizotypal Personality Questionnaire To assess predisposition to Schizotypal Personality Disorder in 257 the neurotypical population, we used the Schizotypal Personality Questionnaire (SPQ) developed by Raine 


\section{Running head: SELF-RECOGNITION OF ACTIONS}

(1991). The 74-item survey was based on criteria from the DSM-III-R (American Psychiatric Association, 1987) to measure schizotypy from multiple dimensions (positive, negative, disorganized, and paranoia) and capture its phenotype, etiology, symptomatology (Wuthrich and Bates, 2006; Barron et al., 2015). We opted for the full-scale SPQ (74 items) rather than the more recent SPQ-brief (32 items; Cohen et al., 2010), as the original SPQ has been shown to provide a clearer division of the individual subscales (Ford and Crewther, 2014), important when considering the reflected overlap between AQ and SPQ subscales.

The SPQ adopts a three-factor structure (analogous to the symptom structure in Schizotypal Personality Disorder and SSC), measuring three main constructs of schizotypy: the cognitive-perceptual dimension (positive schizotypy), interpersonal dimension (negative schizotypy), and disorganized feature dimension (disorganized schizotypy) based on DSM-III-R criteria (Raine, 1994), but well-matched to current DSM$V$ criteria (Barron et al., 2015). Further divisions within the three-factor structure include nine different subscales of the SPQ: ideas of reference, unusual perceptual experiences, odd/magical beliefs, suspiciousness/paranoid ideation (cognitive-perceptual); social anxiety, no close friends, constricted affect (interpersonal); odd behavior and appearance, and odd speech (disorganized) (Raine 1991; 1994). Response criteria on the questionnaire requires binary selection of "true" or "false" (two-point scale) to particular statements (e.g., "I am aware that people notice me when I go out for a meal or to see a film"). Top $10 \%$ of scorers reflect scores greater than 41 , while the bottom $10 \%$ typically score 12 or lower.

Vividness of Motor Imagery Questionnaire The VMIQ-2 (Roberts, 2008) measures introspective reports of vividness of imagery in kinesthetic (movement simulation), internal (first person simulation), and external (third person simulation) visual imagery of 12 different actions (e.g., kicking). Vividness of motor imagery is rated on a five-point Likert scale for each of the 12 actions in each of the three sub-areas. Note that lower scores in VMIQ-2 indicate more vivid images and stronger motor imagery ability. 
Running head: SELF-RECOGNITION OF ACTIONS

\section{Results}

\section{Impact of action types on self-recognition performance}

As shown in Figure 3, participants were able to recognize self-actions significantly above chance performance $(0.25$ in a $4 \mathrm{AFC}$ task): for simple actions with verbal instruction $(M=0.41, S D=0.16)$, $t(100)=9.45, p<.001$, cohen's $d=.940$, for complex actions with verbal instruction $(M=0.54, S D=$

$0.17), t(100)=16.86, p<.001, d=1.678$, and for imitated actions without verbal instruction $(M=0.39$,

$S D=0.16), t(100)=9.20, p<.001, d=.916$, corroborating prior research (e.g., Burling et al., 2019; Loula

et al., 2005). Confidence data showed consistent results with self-recognition accuracy for the action types (see supplementary materials for further detail).

A two-way repeated-measures ANOVA with action orientations and action types as withinsubjects factors on self-recognition performance revealed no effect of action orientation, facing left: $225^{\circ}(M=0.45, S D=0.16)$, front: $0^{\circ}(M=0.45, S D=0.14)$, right: $45^{\circ}(M=0.44, S D=0.15), F(2,200)$

$294=1.58, p=.207, \eta_{\mathrm{p}}^{2}=.016$. The finding is consistent with a previous study showing that self-recognition 295 of walking actions is independent of the viewing angle (Jokisch, Daum, \& Troje, 2004). However, the 296 analysis yielded a significant main effect of action type (simple, complex, and imitation), $F(2,200)=$ $29743.09, p<.001, \eta_{\mathrm{p}}{ }^{2}=.301$. Bonferroni-corrected pairwise comparisons further revealed that self298 recognition was more accurate for complex than simple actions $(t(100)=7.86, p<.001, d=.782)$ and 299 imitation actions $(t(100)=8.56, p<.001, d=.851)$. No difference emerged between simple and imitation 300 actions $(t(100)=0.54, p=.593, d=.053)$. We also found a significant two-way interaction between 301 orientation and action type (Figure 3$), F(4,400)=2.77, p=.027, \eta_{\mathrm{p}}{ }^{2}=.027$, mainly driven by the weaker 302 performance in the frontal view than side views for the imitation actions $\left(F(2,200)=4.124, p=.018, \eta_{\mathrm{p}}{ }^{2}=\right.$ 303 .040). However, the small effect size calls for caution in interpretation of this interaction effect, as the 304 degree of visual ambiguity due to body occlusion likely impacted self-recognition performance for certain 305 action orientations. Among the imitation actions, three actions (e.g., bend, digging hole and chopping 
wood) consisted of the most self-occlusion from the frontal view, resulting in grouping ambiguity in the

307 point-light display.

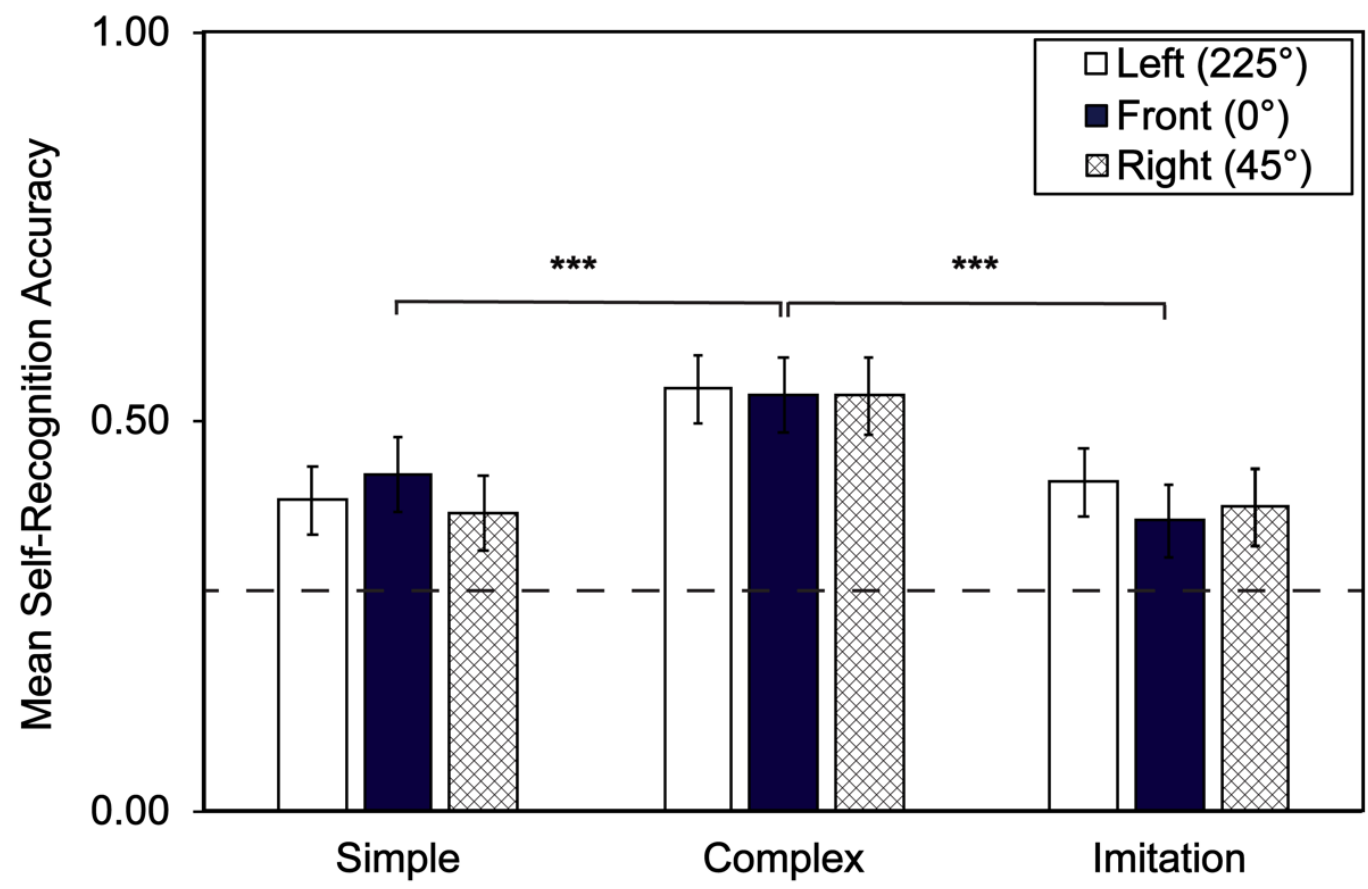

310 Figure 3. Self-recognition accuracy by action types (x-axis) and orientation (colored bars). All action types recognized significantly above chance. Complex actions showed greater self-recognition performance than did simple or imitation actions. Dashed line indicates chance performance (0.25). The error bars indicate standard error of means.

Analysis of item-level variability in self-recognition performance for individual actions (Figure 4)

316 revealed that all complex actions (stretch, get attention, wash windows, argue, guitar, hurry up, fight,

317 baseball, and macarena) were self-recognized significantly above chance $(p s<.001)$. Individual simple

318 actions (point, punch, lift, grab, push, hammer, jump, and kick) except wave, and imitation actions

319 (basketball, conversation, directing traffic p1, directing traffic p2, chopping, digging, and laugh) except

320 bend, were also self-recognized significantly above chance performance $(p s<.030)$.

Due to variability in performance at the individual action level, we further ran an analysis with a

322 linear mixed effects model, including "individual actions" and "participants" as random effects and

323 "action type" as a fixed effect. The model accounted for residual variance in the individual actions across 
Running head: SELF-RECOGNITION OF ACTIONS

324 participants, with a significant main effect of action type on self-recognition performance $(F(2,24)=$ $32511.23, p<.001)$, confirming the impact of the extrinsic factor based on action type.

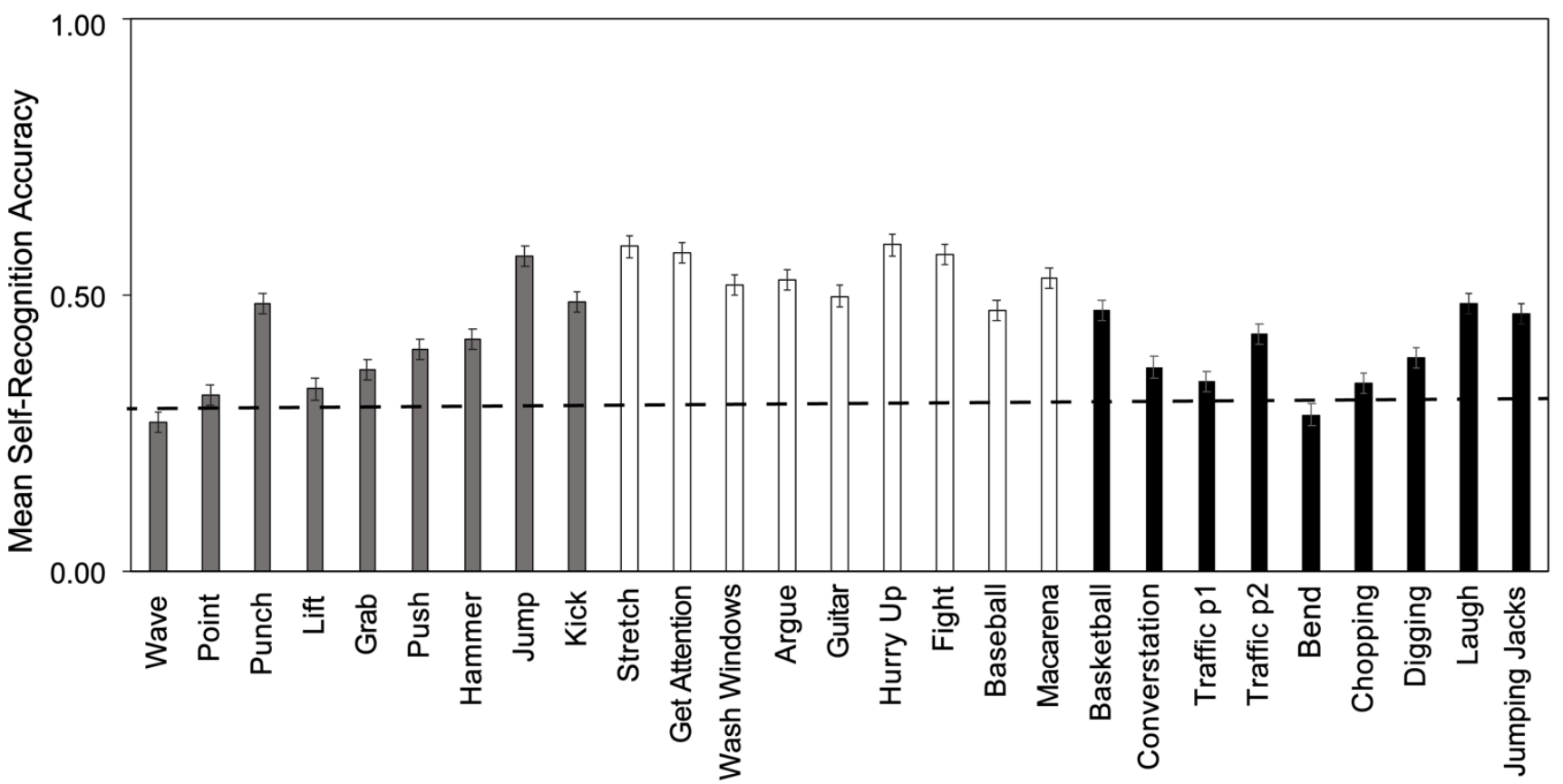

328

329

330

331

332

333

334

335

336

337

338

339

340

341

Figure 4. Mean self-recognition accuracy as a function of the individual action. Color-coding of bars corresponds to action type: simple actions denoted in gray, complex actions denoted in white, imitation actions denoted in black. Dashed line indicates chance performance (0.25). Error bars denote standard error of the means.

To examine the extent that perceptual variability impacted self-recognition accuracy for the extrinsic action factor, we employed an analysis based on similarity measures of motion trajectories in actions, the dynamic time warping (DTW) method. DTW analysis utilizes a non-linear, monotonic warping algorithm to measure the similarity between time series data such as movement trajectories of joints (Gavrila \& Davis, 1995). As DTW measures differences between movement trajectories from actions performed by different people, smaller DTW distance values indicate greater similarity of movement trajectories across participants when they performed the same action. For each participant, we extracted the 3D positions of each of the 17 joints using the BioMotion toolbox (van Boxtel \& Lu, 2013) 


\section{Running head: SELF-RECOGNITION OF ACTIONS}

342 and centered the trajectories to zero for each joint. We then used the DTW function in MATLAB to 343 compute pairwise distances of the joint trajectories across participants. The analysis included the joints of 344 head, neck, clavicle, center/left/right waist, left/right shoulder, stomach, left/right elbow, left/right wrist, 345 left/right knee, left/right ankle for each of the nine simple and nine complex actions. For each participant, 346 a mean distance for an action was computed by averaging across pairwise DTW distances between this 347 participant with all other participants, and then averaged across joints for this action. Two analyses were 348 conducted using the mean DTW distances. First, we found that the correlation between each participant's 349 mean DTW distance and average self-recognition performance for simple and complex actions was non350 significant ( $\operatorname{spearman} \rho=.120,95 \%$ CI $[-.078, .309], p=.233$ ), ruling out the possibility that self351 recognition performance is primarily determined by the variability of body movements of individuals who 352 perform actions in different ways from others. In addition, we computed two mean DTW distance values 353 for simple actions and complex actions respectively for each participant. T-tests on the mean distance 354 values for the action types showed that movement trajectories for complex actions $(M=3.79, S D=1.27)$ 355 were significantly more variable than for simple actions $(M=2.25, S D=0.60), t(100)=-15.80, p<.001$, $356 d=1.57$, consistent with the observation that visual variability generally mapped onto motor planning 357 complexity for the action types.

motor experience (kinesthetic, proprioceptive, and muscle contraction information), but impoverished

361 visual experience from a third-person perspective. By contrast, expertise of movement patterns in many 362 situations, such as sports, show both superior motor ability in performance of one's own actions, but also 363 superior visual ability in recognition of actions performed by others, such as teammates (Hohmann et al., 364 2011). Our task manipulation allowed for the comparison between actions that participants performed 365 (i.e., own imitation action) versus actions that the participant observed (i.e., stick figure imitation actor). 
Running head: SELF-RECOGNITION OF ACTIONS

As shown in Figure 4, participants performed at chance level for visual recognition of previously viewed actions $(M=0.23, S D=0.19), t(72)=-0.718, p=.475, d=-.084$, but showed much greater recognition performance in identification of one's own action $(M=0.38, S D=0.15)$, with visual recognition performance significantly lower than performance in self-identification, $t(72)=5.41, p<.001, d=.633$. The around-chance performance for actions with only visual experience confirms that prior visual experience alone may not suffice for supporting self-recognition. Identifying oneself relies on the ability to simulate the action onto one's own motor system, with visual self-recognition in turn dependent on a matching process to compare simulated action to observed action.

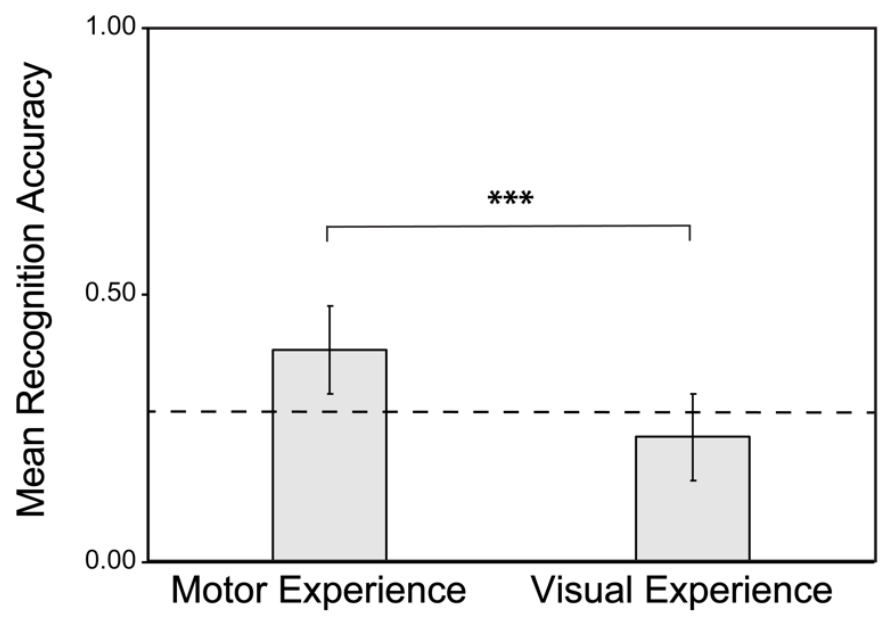

Figure 4. Recognition accuracy for imitation actions in the self-recognition task with motor experience versus performance in the visual-recognition task with visual experience. Significantly weaker performance from recognizing actions from visual experience than for self-recognition from performed actions. Dashed line indicates chance performance (0.25). Error bars indicate standard error of means.

\section{Impact of intrinsic traits on self-recognition performance}

The second analysis step focused on the impact of intrinsic variability in individual difference measures on self-action recognition. Two participants did not complete the VMIQ-2, and one participant did not complete the AQ questionnaire. Hence, listwise deletion resulted in 98 participants included in the individual differences analysis. Descriptive statistics for the scores of autistic traits (AQ score), 
Running head: SELF-RECOGNITION OF ACTIONS

386 schizotypal traits (SPQ score) and motor imagery ability (VMIQ-2 score), and subscale scores are reported 387 in supplementary materials Table 1. Independent sample t-tests revealed no significant effects of sex on 388 composite AQ $(t(96)=1.06, p=.293, d=.239)$, SPQ $(t(96)=0.01, p=.994, d=.002)$, or VMIQ-2 $389(t(96)=-0.98, p=.328, d=-.222)$ scores.

390 We first conducted regression analyses to examine relations between composite scores of 391 individual difference measures and self-recognition performance. For each action type (simple, complex, 392 and imitation), self-recognition performance was set as the dependent variable and three composite scores 393 (AQ for autistic traits, SPQ scores for schizotypal traits, and VMIQ-2 scores for motor imagery ability) 394 were predictor variables in the regression analysis. None of the three regression models revealed 395 significant relationships. However, nonparametric correlation analyses revealed that composite motor 396 imagery (VMIQ-2) scores negatively correlated with self-recognition performance for imitation actions 397 (spearman $\rho=-0.221,95 \%$ CI [-.406, -.017], $p=.029$ ) (see Figure 5). Note that lower VMIQ-2 scores 398 indicate stronger motor imagery ability. Thus, the negative correlation between motor imagery scores and 399 self-recognition performance in imitation condition indicates that individuals with better motor imagery 400 ability showed greater self-recognition performance for imitation actions.

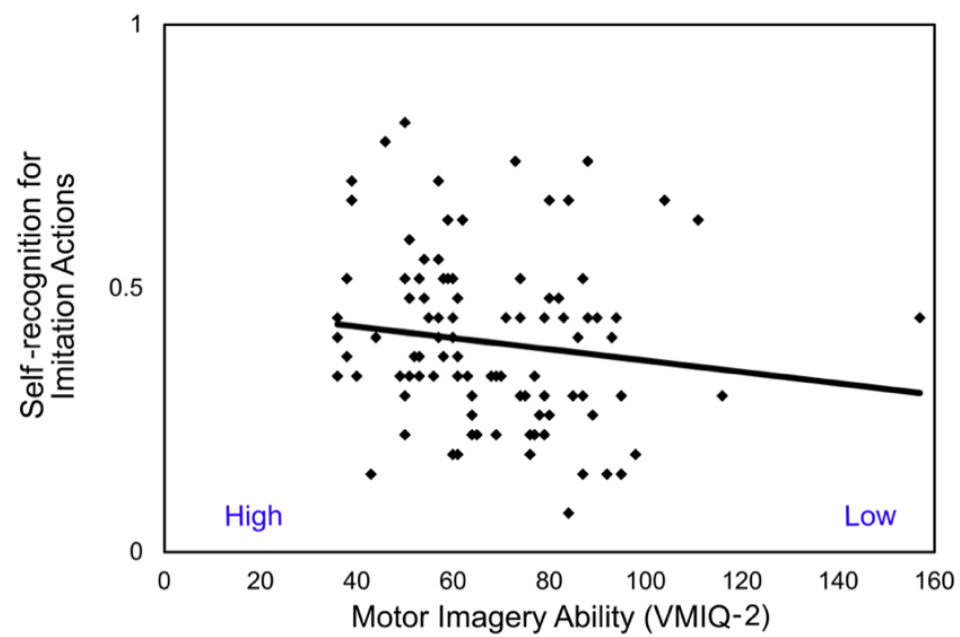

402 Figure 5. Relations between composite motor imagery ability and self-recognition for imitation actions. 403 Note that lower VMIQ-2 score indicates better motor imagery ability. 
Running head: SELF-RECOGNITION OF ACTIONS

Next we examined whether subscale scores in individual difference measures showed relationships with self-recognition performance. We focused on subscale scores for two reasons. First, both ASC and SSC are characterized by multidimensional symptom expression, which may be masked by the aggregate measure. Second, selected subscale scores in autistic traits and schizotypal traits have been shown to relate to atypical biological motion perception (e.g., Blain et al., 2017; Puglia and Morris, 2017) and self409 processing (e.g., Ferroni et al., 2020; Thakkar et al., 2011; Lenzenweger, 2010).

To select important predictor variables from 17 subscale scores ( 5 subscales in AQ, 9 subscales in

411 SPQ and 3 subscales in motor imagery VMIQ-2), we ran three stepwise regressions in relation to self412 recognition performance for the three action types. For the simple action condition, the stepwise regression 413 selected a model with four subscale scores as predictor variables (AQ social communication, and three 414 SPQ subscale scores including odd behavior, ideas of reference, and no close friends) that showed 415 significance in predicting self-recognition performance for simple actions $(F(4,93)=2.817, p=0.030)$. 416 However, among the four selected subscale scores, only AQ social communication scores revealed 417 significant coefficient $(t(92)=-2.672, p=.009)$. Consistently, nonparametric correlation analysis showed 418 a negative relation between AQ social communication and self-recognition for simple actions (spearman $419 \rho=-.229,95 \%$ CI $[-0.414,-0.026], p=.023$ ) (see Figure 6). As higher AQ scores indicate more autistic 420 traits, the negative correlation revealed that participants with more autistic traits in social communication 421 performed worse in self-recognition for simple actions. 


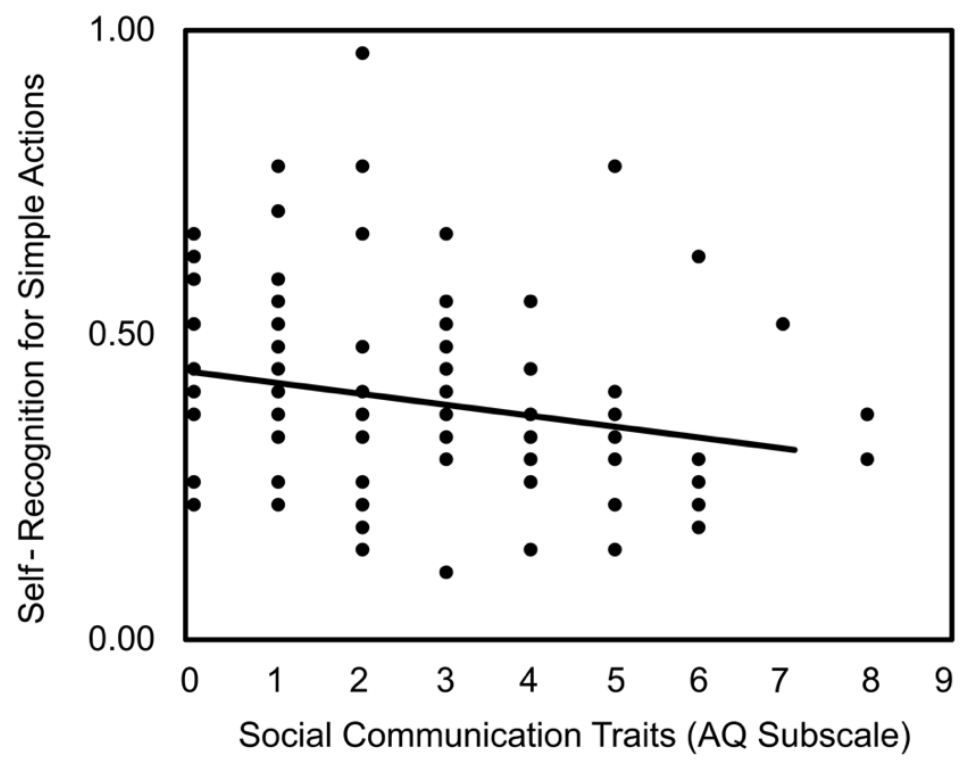

423 Figure 6. Relations between AQ social communication subscale scores and self-recognition for simple scores as predictor variables (including unusual perceptual experiences, odd behavior, ideas of reference and no close friends) in relation to self-recognition performance. But the selected model did not reach statistical significance $(F(3,94)=2.283, p=0.084)$. Upon further examination, we noticed a nonlinear quadratic relationship between SPQ unusual perceptual experience scores and self-recognition for 431 complex actions. Thus, we introduced an additional predictor variable of quadratic SPQ subscale scores 432 of unusual perceptual experiences to the stepwise regression analysis. The results converged to a 433 significant model with two predictor variables (SPQ unusual perceptual experience ssubscale scores, and 434 its quadratic term) $F(2,95)=5.782, p=0.004$. As shown in Figure 7, there was a significant relationship 435 between the SPQ unusual perceptual experiences subscale and self-recognition performance for complex 436 actions (spearman $\rho=0.264,95 \%$ CI $[.064, .445], p=.008)$. In addition to the linear relation, self437 recognition performance for complex actions also related to a quadratic trend of SPQ unusual perceptual 438 scores, revealed by significant coefficients to the quadratic term $(t(92)=-2.937, p=.004)$. The coexistence 
439 of linear and quadratic relations indicates that participants with mid-range SPQ unusual perceptual experiences scores showed best self-recognition performance for complex actions.

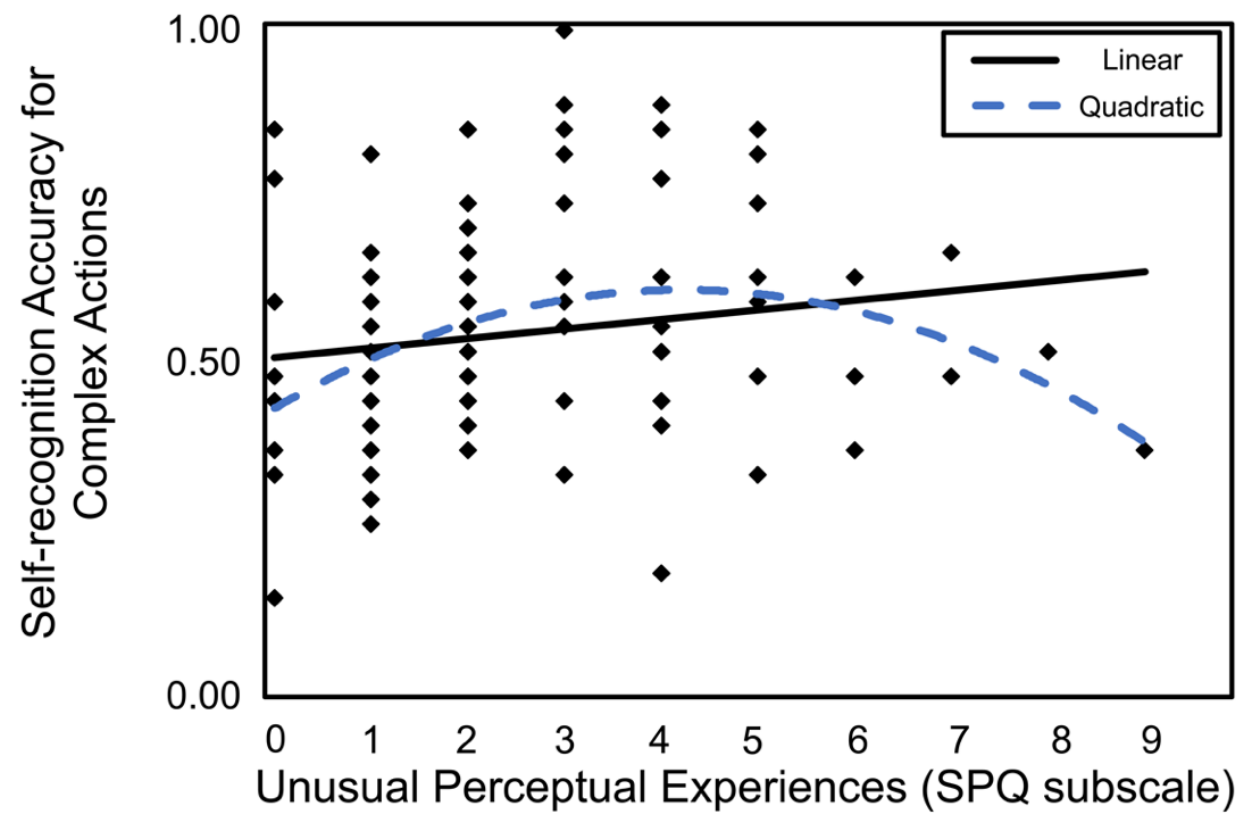

Figure 7. Relations between SPQ unusual perceptual experience subscale scores and self-recognition for complex actions. Note that higher SPQ scores indicate more schizotypal traits.

For imitation actions, the stepwise regression analysis selected a model with six predictor variables, including AQ social communication and attention subscale scores, three SPQ scores of odd speech, suspiciousness, and no close friends, and VMIQ-2 Kinesthetic scores, in relation to selfrecognition performance $(F(6,91)=2.895, p=0.013)$. However, only AQ social communication and $S P Q$ suspiciousness scores showed significant coefficients $(t(90)=-3.286, p=.001 ; t(90)=-2.336, p=.022)$. The follow-up nonparametric correlation analysis did not reveal significant relation between selfrecognition performance and SPQ suspiciousness scores $(p=.263)$, but confirmed its significant relation with the AQ social communication subscale scores (spearman $\rho=-0.229,95 \%$ CI $[-0.414,-0.026], p=$ .023). This result suggests that people with higher AQ traits associated with social communication ability performed worse in the self-recognition task for imitation actions. As self-recognition performance with imitation actions also correlated with the VMIQ-2 composite scores of motor imagery ability as reported 


\section{Running head: SELF-RECOGNITION OF ACTIONS}

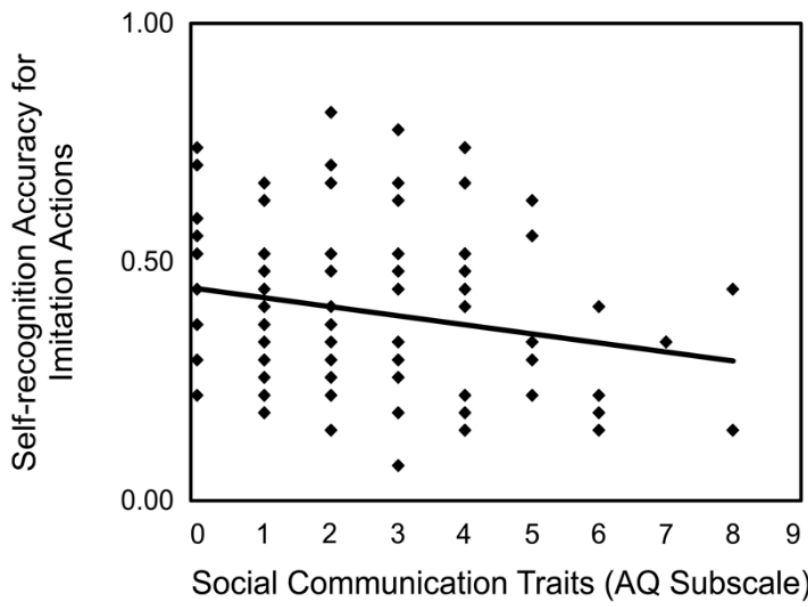

71

early, we ran a second stepwise regression analysis with four prediction variables, including AQ social communication scores and three VMIQ-2 subscale scores. This stepwise regression selected a model with two predictor variables, AQ social communication scores and VMIQ-2 kinesthetic motor imagery scores $(F(2,95)=3.652, p=.030)$. The follow-up nonparametric correlations confirmed the significant relations between self-recognition performance with AQ social communication and kinesthetic VMIQ-2 scores (spearman $\rho=-0.229,95 \%$ CI $[-0.406,-0.017], p=.029)$ as shown in Figure 8.

We further examined how autistic traits and motor imagery abilities jointly impact the selfrecognition performance. A moderation analysis revealed that the negative relationship between AQ social communication scores with self-recognition performance for imitation actions was moderated by kinesthetic motor imagery ability $(\beta=-0.032,95 \%$ CI $[-0.061,-0.003], p=.028)$ : more AQ social communication traits attenuated self-recognition performance, only when kinesthetic motor imagery ability was weakened (-1 SD low: $\beta=-.002,95 \%$ CI $[-0.045,0.042]$; or average: $\beta=0.033,95 \%$ CI [0.065, -0.003], $p=.031)$. Higher kinesthetic motor imagery ability $(+1 S D)$ appeared to provide a compensatory effect, and mitigate the negative impact of high AQ social communication traits on selfrecognition performance with imitation actions $(\beta=-0.066,95 \% \mathrm{CI}[-0.106,-0.025], p=.002)$.

Figure 8. Relations between self-recognition for imitation actions with AQ social communication subscale scores (left panel) and with kinesthetic motor imagery ability (right panel). Note that higher AQ scores indicate more schizotypal traits, and higher VMIQ-K scores indicate weaker imagery ability. 


\section{Running head: SELF-RECOGNITION OF ACTIONS}

\section{Discussion}

On a large sample of participants, we measured the impact of intrinsic traits and extrinsic action factors on self-recognition. Here, we not only replicated previous findings that humans can recognize their own actions in point-light displays (Burling et al., 2019; Loula et al., 2005; Cutting \& Kozlowski, 1977; Prasad and Shiffrar, 2009), but importantly - extended the literature to introduce novel interactions between intrinsic factors (participant traits linked to motor simulation and subclinical sensorimotor processing: ASC and SSC) and extrinsic factors based on the action types (motor planning, action orientation and perceptual variability). The pattern of results further revealed hidden variance within subclinical traits. Specifically, the AQ social communication and SPQ unusual perceptual experiences subscales related to distinct extrinsic action types in self-recognition performance.

\section{Extrinsic variability modulates self-recognition performance}

First, we found that self-recognition performance varied according to the degree of motor planning, delineated by our extrinsic factor of action type (simple, complex, and imitation). A few prior studies have focused on item-level delineations based on perceptual variability or idiosyncrasies (e.g., Loula et al., 2005). However, the perceptual consequences of self-actions are generated from privileged access of one's motor plan during action performance (Knoblich and Prinz, 2001), and hence causally vary as a function of the degree of motor planning (e.g., Li et al., 2020). Complex actions required the greatest degree of motor planning due to the more complex variability of the goal representation space (relative to simple actions) (e.g., Li et al., 2020), and imitation actions required the least motor planning due to mimicry of movement patterns (Zimmer \& Engelkamp, 1996; Schwartz \& Plass, 2014). The performance pattern mostly mapped onto the motor planning differences, with greatest self-recognition performance for complex actions as compared to simple or imitation actions. However, we found no performance difference between simple (simple actions with semantic label; i.e., greater motor planning) and imitation (without semantic label; i.e., reduced motor planning) actions. Although reduced memory recall for 


\section{Running head: SELF-RECOGNITION OF ACTIONS}

499 imitated action labels in comparison to self-generated actions has previously been shown, and attributed

500 to lessened motor planning (see enactment effect; Zimmer and Engelkamp, 1996), the different outcome

501 measures in our task — visual recognition versus enactment (memory recall for actions)—make it difficult

502 to draw conclusive parallels. Recently, a similar study to ours used recognition of point-light actions as

503 the outcome measure (Bläsing and Sauzet, 2018) and also found comparable performance for both

504 imitated and self-generated dancing sequences of unfamiliar actions, significantly greater than the action

505 observation condition. Hence, it is likely that the reduced variability of simple actions degraded the

506 familiarity of the actions, reflected in lower performance than complex actions and comparable

507 performance to imitation actions.

508 What mechanisms underlie self-recognition across action types? Prior work has speculated on two 509 main contributors to self-recognition of actions. First, proprioceptive and kinesthetic information retained 510 from executing familiar motor actions (i.e., motor experience) may principally modulate self-action

511 recognition. On the other hand, participants may recognize their movements on the basis of idiosyncratic 512 visual cues, measured via perceptual variability (i.e., speed of movements; motion trajectories in observed 513 actions). For simple and complex actions, quantitative similarity analyses on both individual actions and 514 individual participants revealed no significant relationship between perceived movement variance and 515 self-recognition accuracy, but showed greater variability in complex than simple actions. For imitation 516 actions, analysis of the visual recognition task showed significantly lower (i.e., chance) performance for 517 recognition of briefly observed visual actions (actor-generated) as compared to self-generated actions. 518 Across all action types, the self-recognition task further demonstrated the viewpoint-invariance (frontal 519 and right/left half-profile) of identifying own actions, regardless of the degree of motor planning, 520 confirming prior findings (Jokisch, Daüm, and Troje, 2006). Together, these findings underscore the 521 importance of the motor system to self-recognition: perceptual experience does not appear to suffice for 522 self-recognition. In keeping with common coding theory, actions signaling a closer match between the 


\section{Running head: SELF-RECOGNITION OF ACTIONS}

523 motor system and perception (i.e., self-generated) enhance action recognition more so than those distally

524 matched (i.e., other-generated) (Prinz, 1997).

\section{Intrinsic interactions with extrinsic factors influence self-recognition performance}

526 Our analyses revealed distinct interactions between the extrinsic factor of action types and

527 intrinsic traits, linked to motor imagery ability and clinical subscales (AQ social communication, and

528 SPQ unusual perceptual experiences). For imitation actions, both composite motor imagery ability and

529 the kinesthetic subtype were significantly correlated with self-recognition performance. Motor

530 simulation theories (Jeannerod 2001; Jeannerod and Pacherie, 2004) posit the central role of motor

531 imagery during action perception. Our results suggest that a compensatory increase in motor imagery

532 ability may be required to self-identify one's own imitation action, which consisted of the least amount

533 of motor planning since no explicit action label was provided during the action execution session. No

534 significant relationships were found between the visual imagery subtypes (internal and external) and

535 self-recognition for any action types. This behavioral dissociation between visual and kinesthetic

536 imagery neatly converges with neural evidence revealing partially distinct brain regions for visual

537 imagery (parieto-occipital) and kinesthetic imagery (i.e., parietal and premotor) (Guillot et al., 2009). It

538 is likely that during the perception of one's own action, motor expertise of own actions makes use of

539 particular neural resources linked to kinesthetic motor imagery (e.g., Wei and Luo, 2010; Kim et al., 540 2011).

541 We also found negative relationships between the AQ social communication subscale and self542 recognition performance for simple and imitation actions, but not for complex actions. This negative 543 relationship was moderated by kinesthetic motor imagery traits: greater AQ social communication traits 544 attenuated self-recognition performance for imitation actions, only when kinesthetic motor imagery 545 ability was weakened (low or average). In other words, higher kinesthetic motor imagery ability 546 appeared to mitigate the AQ social communication self-action performance difference. As motor 


\section{Running head: SELF-RECOGNITION OF ACTIONS}

547 imagery is presumed to share neural resources with biological motion perception (e.g., Grèzes and

548 Decety 2001) the negative AQ social communication relation may be driven by difficulties in biological

549 motion processing, consistent with reduced brain activity in prefrontal and left temporal cortices in high

550 AQ social communication individuals when viewing point-light actions (Puglia and Morris, 2017). This

551 interpretation dovetails a recent behavioral study linking the AQ social communication subscale to

552 atypical processing of local kinematic information in biological motion (Wang et al., 2018). For simple

553 actions with less perceptual variability, superior local processing may be necessary to discriminate fine

554 details of self from other actions, possibly compromised in those with high AQ social communication

555 traits. By consequence, the nonsignificant relationship between AQ social communication and complex

556 actions may result from the increased perceptual variability in the actions resulting from individualized

557 body movements, providing a compensatory strategy to self-recognition, regardless of the AQ spectrum.

558 Notably, the interpretation of weakened local processing in high AQ social communication traits is in

559 contrast to behavioral patterns observed in biological motion research that utilize the composite AQ

560 measure. Many of these studies show the reverse pattern — superior local processing and a generalized

561 disturbance in global processing in high AQ individuals. However, findings from these studies remain

562 inconsistent (largely attributed to task-specific variability) (e.g., van Boxtel et al., 2017) and a possible

563 reconciliation could be obtained through exploration of the subscales, rather than on the aggregate

564 measures.

565 Scores on SPQ unusual perceptual experiences were quadratically related to complex actions,

566 such that low and high individuals performed worse at self-recognizing complex action sequences. How

567 might the intrinsic SPQ subscale modulate performance on complex actions? Prior work suggests

568 impaired action perception across the schizophrenia spectrum (Kim et al., 2005, Kim et al., 2011).

569 However, we only observed a significant trend with self-recognition performance on one action type

570 (i.e., complex actions). Thus, the present results cannot be reduced to a generalized abnormality in 


\section{Running head: SELF-RECOGNITION OF ACTIONS}

571 biological motion perception. Rather, atypical action monitoring in suppressing the sensory

572 consequences of action may produce false positives - misattributions that the action is externally

573 generated (Frith et al., 2000). The unusual perceptual subscale focuses on the positive (i.e., first-rank

574 symptoms) of SSC, including incoherent perceptual experiences that reduce one's sense of agency (Asai

575 et al., 2008), blurring the boundaries between the self and other (e.g., Waters \& Badcock, 2010; Postmes

576 et al., 2014). In relation to atypical action monitoring, prior studies have shown that individuals high in

577 SPQ unusual perceptual traits are more successful at self-induced tickling (i.e., Lemaitre, Luyat, \&

578 Lafargue, 2016), likened to a disrupted match between predicted efference copies and sensorimotor

579 outcomes; see forward model accounts (Wolpert and Miall, 1996; Postmes et al., 2014). The action

580 monitoring atypicality may be particularly exacerbated by complex actions that require a greater degree

581 of motor planning and preparation. Weakened self-recognition performance on complex actions with

582 those low on SPQ unusual perceptual experiences traits is less clear. A likely possibility may be due to

583 decreased perceptual acuity in discerning subtle movement patterns, induced by the distraction of

584 perceptual variance in complex action sequences. This could manifest in behavioral differences based on

585 false negatives (i.e., decreased discrimination of perceptual idiosyncrasies), rather than false positives

586 (i.e., misattributions).

587 Neither composite subclinical measure (schizotypal or autistic) showed a relation to self-

588 recognition performance. If our analysis was restricted to the composite measures, this would suggest

589 that physical aspects of the self (e.g., bodily recognition) are intact in both conditions, consistent with

590 many studies on visual self-recognition (e.g., Ferrari \& Matthews, 1983; Uddin et al., 2008; Dunphy-

591 Lelii \& Wellman, 2012; Gillespie-Smith et al., 2014; Lee et al., 2007; cf. Chakraborty and Chakrabarti,

592 2015). However, the clear pattern of relations with the subscales suggests that a focus on the composite

593 measure can be misleading, as it masks the psychopathological variance in the general population. SSC

594 and ASC have heterogenous socio-cognitive symptomatology, residing on a continuum that extends well 


\section{Running head: SELF-RECOGNITION OF ACTIONS}

595 into the general population, and frequently updated in light of DSM revisions. While models of AQ and

596 SPQ symptomatology posit the large phenotypic overlap between AQ and SPQ subscales (which our

597 results similarly maintain), the AQ social communication and SPQ unusual perceptual experiences

598 dimensions appear to reside on a diametric axis, largely exempt from the overlap (Dinsdale et al 2013;

599 Ford and Crewther, 2014). The present results confirm the diametric relation presented in the prior work,

600 as we found no relationship between the subdimensions of interest (i.e., AQ social communication and

601 SPQ unusual perceptual experiences), which shared unique variance with each respective clinical

602 composite measure. Since the composite measures average across the spectrum, a foundational approach

603 for future individual difference studies should prioritize subscale parcellations, which may reveal finer-

604 grained individual variability.

605 Our study presents some outstanding limitations. Though we separated actions based on motor

606 planning, our actions included a range of social and non-social actions. In doing so, we preserved the

607 naturalistic nature of the actions; however, additional studies can more specifically isolate the

608 contribution of controlled action types. Another limitation pertained to the movement similarity analysis.

609 Participants may have used different limbs to perform some actions, which may artificially inflate the

610 dissimilarity. Additionally, subtle movement differences, not capturable with dynamic time warping

611 analysis across groups, may also be needed to see whether motor performance differences are associated

612 with psychometrically assessed ASC and schizotypal traits (e.g., Walker et al., 1999; Lenzenweger \&

613 Maher, 2002). Other quantitative metrics of variability (e.g., Coste et al., 2020) on these action types

614 would also be beneficial, while out of scope for the present paper.

Few species are capable of self-recognition and develop motor-based, self-other mapping

616 mechanisms in the brain (i.e., the mirror neuron system). Visual self-recognition of point-light actions

617 provides a unique lens to gauge the core perceptual and motor mechanisms underlying self-

618 representations in humans. These paradigms control for the level of visual familiarity people have with 
Running head: SELF-RECOGNITION OF ACTIONS

619 self-related stimuli (e.g., compared to familiar faces, body images, voices) from a third-person

620 perspective, highlighting the importance of "acting" in "seeing" the self. Our findings thus complement

621 a diverse body of literature in self-processing, demonstrating that self-recognition is possible for stimuli

622 even with little visual experience. Furthermore, the approach of studying individual differences provides

623 new evidence for how the motor system may contribute to self-recognition — distinct aspects of motor

624 processing tap into intrinsic and extrinsic routes, imbuing the core of self-awareness.

625

626

627

628

629

630

631

632

633

\section{Acknowledgements}

We thank Tabitha Safari, Nick Ichien, Aya Strauss, Lizeth Sotelo, Justin Azarian, Marian Spannowsky, Sunhee Jin, and Nazar Flome for assistance in data collection. We thank Joseph Burling and Steve Thurman for help with the motion capture system. This study was supported by the National Science Foundation (BCS-1655300).

\section{Open Practices}

This study was not preregistered. 
Running head: SELF-RECOGNITION OF ACTIONS

\section{References}

635

636

637

638

639

640

641

642

643

644

645

646

647

648

649

650

651

652

653

654

655

656

657

658

659

660

661

662

663

664

665

666

667

668

Ahmed, A. A., \& Vander Wyk, B. C. (2013). Neural processing of intentional biological motion in unaffected siblings of children with autism spectrum disorder: An fMRI study. Brain and Cognition. https://doi.org/10.1016/j.bandc.2013.09.007

American Psychiatric Association. (1987). Diagnostic and statistical manual of mental disorders (3rd ed., rev.).

Asai, T., Sugimori, E., \& Tanno, Y. (2008). Schizotypal personality traits and prediction of one's own movements in motor control: What causes an abnormal sense of agency? Consciousness and Cognition, 17(4), 1131-1142. https://doi.org/10.1016/j.concog.2008.04.004

Asperger, H. (1944). Die "autistichen Psychopathen" im Kinder- salter. Archive fur psychiatrie und Nervenkrankheiten, 117, 76- 136. (Reprinted in Autism and Asperger syndrome, by U. Frith, Ed., 1991, Cambridge, UK: Cambridge University Press).

Binet, A. (1886). La Psychologie du Raisonnement. In Alcan (Ed.). Paris

Baron-Cohen, S., Wheelwright, S., Skinner, R., Martin, J., \& Clubley, E. (2001). The autism-spectrum quotient (AQ): evidence from Asperger syndrome/high-functioning autism, males and females, scientists and mathematicians.[erratum appears in J Autism Dev Disord 2001 Dec;31(6):603]. Journal of Autism \& Developmental Disorders.

Barron, D., Swami, V., Towell, T., Hutchinson, G., \& Morgan, K. D. (2015). Examination of the factor structure of the schizotypal personality questionnaire among British and Trinidadian adults. BioMed Research International. https://doi.org/10.1155/2015/258275

Beardsworth, T., \& Buckner, T. (1981). The ability to recognize oneself from a video recording of one's movements without seeing one's body. Bulletin of the Psychonomic Society. https://doi.org/10.3758/BF03333558

Bekkering, H., Wohlschläger, A., \& Gattis, M. (2000). Imitation of gestures in children is goal-directed. Quarterly Journal of Experimental Psychology Section A: Human Experimental Psychology, 53(1), 153-164. https://doi.org/10.1080/713755872

Bischoff, M., Zentgraf, K., Lorey, B., Pilgramm, S., Balser, N., Baumgartner, E., Hohmann, T., Stark, R., Vaitl, D., \& Munzert, J. (2012). Motor familiarity: Brain activation when watching kinematic displays of one's own movements. Neuropsychologia. https://doi.org/10.1016/j.neuropsychologia.2012.05.009

Blain, S. D., Peterman, J. S., \& Park, S. (2017). Subtle cues missed: Impaired perception of emotion from gait in relation to schizotypy and autism spectrum traits. Schizophrenia Research, 183(September 2017), 157-160. https://doi.org/10.1016/j.schres.2016.11.003

Blake, R., \& Shiffrar, M. (2007). Perception of human motion. In Annual Review of Psychology. https://doi.org/10.1146/annurev.psych.57.102904.190152 
Running head: SELF-RECOGNITION OF ACTIONS

669

670

671

672

673

674

675

676

677

678

679

680

681

682

683

684

685

686

687

688

689

690

691

692

693

694

695

696

697

698

699

700

701

702

Bläsing, B. E., \& Sauzet, O. (2018). My action, my self: Recognition of self-created but visually unfamiliar dance-like actions from point-light displays. Frontiers in Psychology, 9(OCT), 1-9. https://doi.org/10.3389/fpsyg.2018.01909

Bleuler, E. Dementia Praecox: Or the Group of Schizophrenias. New York: International Universities Press; 1911.

Borda, J. P., \& Sass, L. A. (2015). Phenomenology and neurobiology of self disorder in schizophrenia: Primary factors. Schizophrenia Research, 169(1-3), 464-473. https://doi.org/10.1016/j.schres.2015.09.024

Bralten, J., Van Hulzen, K. J., Martens, M. B., Galesloot, T. E., Arias Vasquez, A., Kiemeney, L. A., Buitelaar, J. K., Muntjewerff, J. W., Franke, B., \& Poelmans, G. (2018). Autism spectrum disorders and autistic traits share genetics and biology. Molecular Psychiatry, 23(5), 1205-1212. https://doi.org/10.1038/mp.2017.98

Burling, J. M., Kadambi, A., Safari, T., \& Lu, H. (2019). The impact of autistic traits on self-recognition of body movements. Frontiers in Psychology. https://doi.org/10.3389/fpsyg.2018.02687

Casile, A., \& Giese, M. A. (2006). Nonvisual motor training influences biological motion perception. Current Biology. https://doi.org/10.1016/j.cub.2005.10.071

Chakraborty, A., \& Chakrabarti, B. (2015). Is it me? Self-recognition bias across sensory modalities and its relationship to autistic traits. Molecular Autism, 6(1), 1-8. https://doi.org/10.1186/s13229-0150016-1

Clark, S., Tremblay, F., \& Ste-Marie, D. (2004). Differential modulation of corticospinal excitability during observation, mental imagery and imitation of hand actions. Neuropsychologia. https://doi.org/10.1016/S0028-3932(03)00144-1

Cohen, A. S., Matthews, R. A., Najolia, G. M., \& Brown, L. A. (2010). Toward a more psychometrically sound brief measure of Schizotypal traits: Introducing the SPQ-Brief revised. Journal of Personality Disorders. https://doi.org/10.1521/pedi.2010.24.4.516

Conson, M., Aromino, A. R., \& Trojano, L. (2010). Whose hand is this? Handedness and visual perspective modulate self/other discrimination. Experimental Brain Research, 206, 449-453.

Cook, R., Johnston, A., \& Heyes, C. (2012). Self-recognition of avatar motion: How do I know it's me? Proceedings of the Royal Society B: Biological Sciences. https://doi.org/10.1098/rspb.2011.1264

Coste, A., Bardy, B. G., Janaqi, S., Słowiński, P., Tsaneva-Atanasova, K., Goupil, J. L., \& Marin, L. (2020). Decoding identity from motion: how motor similarities colour our perception of self and others. Psychological Research. https://doi.org/10.1007/s00426-020-01290-8

Cutting, J. E., \& Kozlowski, L. T. (1977). Recognizing friends by their walk: Gait perception without familiarity cues. Bulletin of the Psychonomic Society. https://doi.org/10.3758/BF03337021 
Running head: SELF-RECOGNITION OF ACTIONS

703

704

705

706

707

708

709

710

711

712

713

714

715

716

717

718

719

720

721

722

723

724

725

726

727

728

729

730

731

732

733

734

735

Daprati, E., \& Sirigu, A. (2002). Laterality effects on motor awareness. Neuropsychologia. https://doi.org/10.1016/S0028-3932(01)00203-2

Daprati, E., Wriessnegger, S., \& Lacquaniti, F. (2007). Knowledge of one's kinematics improves perceptual discrimination. Consciousness and Cognition. https://doi.org/10.1016/j.concog.2006.03.001

Dinsdale, N. L., Hurd, P. L., Wakabayashi, A., Elliot, M., \& Crespi, B. J. (2013). How are autism and schizotypy related? Evidence from a non-clinical population. PloS one, 8(5), e63316.

Dunphy-Lelii, S., \& Wellman, H. M. (2012). Delayed self-recognition in autism: A unique difficulty?. Research in Autism Spectrum Disorders, 6(1), 212-223.

Engelkamp, J., \& Krumnacker, H. (1980). Image- and motor-processes in the retention of verbal materials. [Image- and motor-processes in the retention of verbal materials.]. Zeitschrift Für Experimentelle Und Angewandte Psychologie.

Ferrari, M., \& Matthews, W. S. (1983). Self-recognition deficits in autism: Syndrome-specific or general developmental delay? Journal of Autism and Developmental Disorders, 13(3), 317-324. https://doi.org/10.1007/BF01531569

Ferri, F., Frassinetti, F., Ardizzi, M., Costantini, M., \& Gallese, V. (2012). A sensorimotor network for the bodily self. Journal of Cognitive Neuroscience, 24, 1584-1595.

Ferri, F., Frassinetti, F., Costantini, M., \& Gallese, V. (2011). Motor simulation and the bodily self. PLOS ONE, 6, e17927.

Ferroni, F., Ardizzi, M., Ferri, F., Tesanovic, A., Langiulli, N., Tonna, M., Marchesi, C., \& Gallese, V. (2020). Schizotypy and individual differences in peripersonal space plasticity. Neuropsychologia, 147(May), 107579. https://doi.org/10.1016/j.neuropsychologia.2020.107579

Flach, R., Knoblich, G., \& Prinz, W. (2004). Recognizing one's own clapping: the role of temporal cues. Psychological Research. https://doi.org/10.1007/s00426-003-0165-2

Fletcher, P. C., \& Frith, C. D. (2009). Perceiving is believing: a Bayesian approach to explaining the positive symptoms of schizophrenia. Nature Reviews Neuroscience, 10(1), 48-58.

Ford, T. C., \& Crewther, D. P. (2014). Factor analysis demonstrates a common schizoidal phenotype within autistic and schizotypal tendency: Implications for neuroscientific studies. Frontiers in Psychiatry. https://doi.org/10.3389/fpsyt.2014.00117

Frassinetti, F., Pavani, F., Zamagni, E., Fusaroli, G., Vescovi, M., Benassi, M., ... \& Farnè, A. (2009). Visual processing of moving and static self body-parts. Neuropsychologia, 47(8-9), 1988-1993.

Frassinetti, F., Ferri, F., Maini, M., Benassi, M. G., \& Gallese, V. (2011). Bodily self: An implicit knowledge of what is explicitly unknown. Experimental Brain Research, 212, 153-160. 
Running head: SELF-RECOGNITION OF ACTIONS

736

737

738

739

740

741

742

743

744

745

746

747

748

749

750

751

752

753

754

755

756

757

758

759

760

761

762

763

764

765

766

767

768

769

Frith, C. D., Blakemore, S. J., \& Wolpert, D. M. (2000). Explaining the symptoms of schizophrenia: Abnormalities in the awareness of action. Brain Research Reviews. https://doi.org/10.1016/S01650173(99)00052-1

Gallese, V., \& Ferri, F. (2014). Psychopathology of the bodily self and the brain: The case of schizophrenia. Psychopathology, 47(6), 357-364. https://doi.org/10.1159/000365638

Ganesh, S., van Schie, H. T., Cross, E. S., de Lange, F. P., \& Wigboldus, D. H. J. (2015). Disentangling neural processes of egocentric and allocentric mental spatial transformations using whole-body photos of self and other. NeuroImage. https://doi.org/10.1016/j.neuroimage.2015.05.003

Gavrila, D. M., \& Davis, L. S. (1995). Towards 3-D model-based tracking and recognition of human movement: a multi-view approach. Gesture.

Gessaroli, E., Andreini, V., Pellegri, E., \& Frassinetti, F. (2013). Self-face and self-body recognition in autism. Research in Autism Spectrum Disorders. https://doi.org/10.1016/j.rasd.2013.02.014

Gillespie-Smith, K., Ballantyne, C., Branigan, H. P., Turk, D. J., \& Cunningham, S. J. (2018). The I in autism: Severity and social functioning in autism are related to self-processing. British Journal of Developmental Psychology, 36(1), 127-141. https://doi.org/10.1111/bjdp.12219

Grèzes, J., Fonlupt, P., Bertenthal, B., Delon-Martin, C., Segebarth, C., \& Decety, J. (2001). Does perception of biological motion rely on specific brain regions? NeuroImage. https://doi.org/10.1006/nimg.2000.0740

Guillot, A., Collet, C., Nguyen, V. A., Malouin, F., Richards, C., \& Doyon, J. (2009). Brain activity during visual versus kinesthetic imagery: An fMRI study. Human Brain Mapping, 30(7), 21572172. https://doi.org/10.1002/hbm.20658

Hohmann, T., Troje, N. F., Olmos, A., \& Munzert, J. (2011). The influence of motor expertise and motor experience on action and actor recognition. Journal of Cognitive Psychology. https://doi.org/10.1080/20445911.2011.525504

Hur, J. W., Blake, R., Cho, K. I. K., Kim, J., Kim, S. Y., Choi, S. H., Kang, D. H., \& Kwon, J. S. (2016). Biological motion perception, brain responses, and schizotypal personality disorder. JAMA Psychiatry, 73(3), 260-267. https://doi.org/10.1001/jamapsychiatry.2015.2985

Iacoboni, M., \& Dapretto, M. (2006). The mirror neuron system and the consequences of its dysfunction. In Nature Reviews Neuroscience. https://doi.org/10.1038/nrn2024

Jeannerod, M. (2001). Neural simulation of action: A unifying mechanism for motor cognition. NeuroImage. https://doi.org/10.1006/nimg.2001.0832

Jeannerod, M., \& Pacherie, E. (2004). Agency, Simulation and Self-identification. 19(2), 113-146.

Johansson, G. (1973). Visual perception of biological motion and a model for its analysis. Perception \& Psychophysics. https://doi.org/10.3758/BF03212378 
Running head: SELF-RECOGNITION OF ACTIONS

770

771

772

773

774

775

776

777

778

779

780

781

782

783

784

785

786

787

788

789

790

791

792

793

794

795

796

797

798

799

800

801

Johns, L. C., \& Van Os, J. (2001). The continuity of psychotic experiences in the general population. In Clinical Psychology Review. https://doi.org/10.1016/S0272-7358(01)00103-9

Jokisch, D., Daum, I., \& Troje, N. F. (2006). Self recognition versus recognition of others by biological motion: Viewpoint-dependent effects. Perception. https://doi.org/10.1068/p5540

Kanner, L. (1943). Autistic disturbances of affective contact. Nervous child, 2(3), 217-250.

Kennel, C., Hohmann, T., \& Raab, M. (2014). Action perception via auditory information: Agent identification and discrimination with complex movement sounds. Journal of Cognitive Psychology, 26(2), 157-165. https://doi.org/10.1080/20445911.2013.869226

Kim, J., Doop, M. L., Blake, R., \& Park, S. (2005). Impaired visual recognition of biological motion in schizophrenia. Schizophrenia Research. https://doi.org/10.1016/j.schres.2005.04.006

Kim, J., Park, S., \& Blake, R. (2011). Perception of Biological Motion in Schizophrenia and Healthy Individuals: A Behavioral and Fmri Study. PLoS ONE. https://doi.org/10.1371/journal.pone.0019971

Kraeplin, E., 1919. Dementia Praecox and Paraphrenia (R.M. Barclay, G.M. Robertson, Trans.). E and S Livingstone. Edinburgh.

Knoblich, G., \& Flach, R. (2001). Predicting the effects of actions: Interactions of Perception and Action. Psychological Science, 12(6), 467-472. https://doi.org/10.1111/1467-9280.00387

LaViola, J. J. (2003). Double exponential smoothing: An alternative to Kalman filter-based predictive tracking. Proceedings of the Workshop on Virtual Environments, EGVE'03. https://doi.org/10.1145/769953-769976

Lee, J., Kwon, J. S., Shin, Y. W., Lee, K. J., \& Park, S. (2007). Visual self-recognition in patients with schizophrenia. Schizophrenia Research, 94(1-3), 215-220.

Lemaitre, A. L., Luyat, M., \& Lafargue, G. (2016). Individuals with pronounced schizotypal traits are particularly successful in tickling themselves. Consciousness and Cognition. https://doi.org/10.1016/j.concog.2016.02.005

Lenzenweger, M. F., \& Maher, B. A. (2002). Psychometric schizotypy and motor performance. Journal of Abnormal Psychology. https://doi.org/10.1037/0021-843X.111.4.546

Li, X., Krol, M. A., Jahani, S., Boas, D. A., Tager-Flusberg, H., \& Yücel, M. A. (2020). Brain correlates of motor complexity during observed and executed actions. Scientific Reports, 10(1). https://doi.org/10.1038/s41598-020-67327-5

Loula, F., Prasad, S., Harber, K., \& Shiffrar, M. (2005). Recognizing People From Their Movement. https://doi.org/10.1037/0096-1523.31.1.210 
Running head: SELF-RECOGNITION OF ACTIONS

802

803

804

805

806

807

808

809

810

811

812

813

814

815

816

817

818

819

820

821

822

823

824

825

826

827

828

829

830

831

832

833

834

835

Miller, L. E., \& Saygin, A. P. (2013). Individual differences in the perception of biological motion: Links to social cognition and motor imagery. Cognition. https://doi.org/10.1016/j.cognition.2013.03.013

Mitchell, R. W., \& Curry, C. (2016). Self-Recognition and Other-Recognition in Point-Light Displays. Open Journal of Philosophy, 06(01), 42-50. https://doi.org/10.4236/ojpp.2016.61005

Molnar-Szakacs, I., Kaplan, J., Greenfield, P. M., \& Iacoboni, M. (2006). Observing complex action sequences: The role of the fronto-parietal mirror neuron system. NeuroImage. https://doi.org/10.1016/j.neuroimage.2006.07.035

Murgia, M., Hohmann, T., Galmonte, A., Raab, M., \& Agostini, T. (2012). Recognising one's own motor actions through sound: The role of temporal factors. Perception, 41(8), 976-987. https://doi.org/10.1068/p7227

Noel, J. P., Cascio, C. J., Wallace, M. T., \& Park, S. (2017). The spatial self in schizophrenia and autism spectrum disorder. Schizophrenia research, 179, 8-12.

Oberman, L. M., \& Ramachandran, V. S. (2007). The simulating social mind: The role of the mirror neuron system and simulation in the social and communicative deficits of autism spectrum disorders. In Psychological Bulletin. https://doi.org/10.1037/0033-2909.133.2.310

Parnas, J., \& Henriksen, M. G. (2014). Disordered self in the schizophrenia spectrum: A clinical and research perspective. Harvard Review of Psychiatry, 22(5), 251-265. https://doi.org/10.1097/HRP.0000000000000040

Perrykkad, K., \& Hohwy, J. (2020). Modelling Me, Modelling You: the Autistic Self. Review Journal of Autism and Developmental Disorders, 7(1). https://doi.org/10.1007/s40489-019-00173-y

Postmes, L., Sno, H. N., Goedhart, S., van der Stel, J., Heering, H. D., \& de Haan, L. (2014). Schizophrenia as a self-disorder due to perceptual incoherence. Schizophrenia Research, 152(1), 41-50. https://doi.org/10.1016/j.schres.2013.07.027

Prasad, S., \& Shiffrar, M. (2009). Viewpoint and the Recognition of People From Their Movements. Journal of Experimental Psychology: Human Perception and Performance. https://doi.org/10.1037/a0012728

Prinz, W. (1997). Perception and Action Planning. European Journal of Cognitive Psychology. https://doi.org/10.1080/713752551

Puglia, M. H., \& Morris, J. P. (2017). Neural response to biological motion in healthy adults varies as a function of autistic-like traits. Frontiers in Neuroscience, 11(JUL), 1-10. https://doi.org/10.3389/fnins.2017.00404

Raine, A. (1991). The spq: A scale for the assessment of schizotypal personality based on DSM-III-r criteria. Schizophrenia Bulletin. https://doi.org/10.1093/schbul/17.4.555 
Running head: SELF-RECOGNITION OF ACTIONS

836

837

838

839

840

841

842

843

844

845

846

847

848

849

850

851

852

853

854

855

856

857

858

859

860

861

862

863

864

865

866

867

868

869

870

Raine, A., Reynolds, C., Lencz, T., Scerbo, A., Triphon, N., \& Kim, D. (1994). Cognitive-perceptual, interpersonal, and disorganized features of schizotypal personality. Schizophrenia Bulletin. https://doi.org/10.1093/schbul/20.1.191

Repp, B. H., \& Knoblich, G. (2004). Perceiving action identity: How pianists recognize their own performances. Psychological Science. https://doi.org/10.1111/j.0956-7976.2004.00727.x

Rizzolatti, G., Fogassi, L., \& Gallese, V. (2001). Neurophysiological mechanisms underlying the understanding and imitation of action. Nature Reviews Neuroscience. https://doi.org/10.1038/35090060

Roberts, R., Callow, N., Hardy, L., Markland, D., \& Bringer, J. (2008). Movement imagery ability: Development and assessment of a revised version of the vividness of movement imagery questionnaire. Journal of Sport and Exercise Psychology. https://doi.org/10.1123/jsep.30.2.200

Ruzich, E., Allison, C., Smith, P., Watson, P., Auyeung, B., Ring, H., \& Baron-Cohen, S. (2016). Subgrouping siblings of people with autism: Identifying the broader autism phenotype. Autism Research,9(6), 658-665. https://doi.org/10.1002/aur.1544

Sandsten, K. E., Nordgaard, J., Kjaer, T. W., Gallese, V., Ardizzi, M., Ferroni, F., Petersen, J., \& Parnas, J. (2020). Altered self-recognition in patients with schizophrenia. Schizophrenia Research, 218, 116-123. https://doi.org/10.1016/j.schres.2020.01.022

Sass, L. A., \& Parnas, J. (1998). Schizophrenia , Consciousness , and the Self. 427-444.

Schneider, K. (1959). Clinical psychopathology. (Trans. by M. W. Hamilton) (5th ed.). Grune \& Stratton.

Schwartz, R. N., \& Plass, J. L. (2014). Click versus drag: User-performed tasks and the enactment effect in an interactive multimedia environment. Computers in Human Behavior. https://doi.org/10.1016/j.chb.2014.01.012

Sevdalis, V., \& Keller, P. E. (2009). Self-recognition in the perception of actions performed in synchrony with music. Annals of the New York Academy of Sciences. https://doi.org/10.1111/j.1749-6632.2009.04773.x

Siever, L. J., \& Davis, K. L. (2004). The Pathophysiology of Schizophrenia Disorders: Perspectives from the Spectrum. American Journal of Psychiatry, 161(3), 398-413. https://doi.org/10.1176/appi.ajp.161.3.398

Thakkar, K. N., Nichols, H. S., McIntosh, L. G., \& Park, S. (2011). Disturbances in body ownership in schizophrenia: Evidence from the rubber hand illusion and case study of a spontaneous out-of-body experience. PLoS ONE, 6(10). https://doi.org/10.1371/journal.pone.0027089

Thurman, S. M., van Boxtel, J. J., Monti, M. M., Chiang, J. N., \& Lu, H. (2016). Neural adaptation in pSTS correlates with perceptual aftereffects to biological motion and with autistic traits. Neuroimage, 136, 149-161. 
Running head: SELF-RECOGNITION OF ACTIONS

871

872

873

874

875

876

877

878

879

880

881

882

883

884

885

886

887

888

889

890

891

892

893

894

895

896

897

898

899

900

901

902

903

904

905

906

Torbet, G., Schulze, D., Fiedler, A., \& Reuter, B. (2015). Assessment of self-disorders in a non-clinical population: Reliability and association with schizotypy. Psychiatry Research. https://doi.org/10.1016/j.psychres.2015.05.011

van Boxtel, J. J. A., \& Lu, H. (2013). A biological motion toolbox for reading, displaying, and manipulating motion capture data in research settings. Journal of Vision. https://doi.org/10.1167/13.12.7

van Boxtel, J. J. A., Peng, Y., Su, J., \& Lu, H. (2017). Individual differences in high-level biological motion tasks correlate with autistic traits. Vision Research. https://doi.org/10.1016/j.visres.2016.11.005

Viviani, P., \& Stucchi, N. (1992). Biological movements look uniform: Evidence of motor-perceptual interactions. Journal of Experimental Psychology: Human Perception and Performance, 18(3), 603-623. https://doi.org/10.1037//0096-1523.18.3.603

Vollema, M. G., Sitskoorn, M. M., Appels, M. C. M., \& Kahn, R. S. (2002). Does the Schizotypal Personality Questionnaire reflect the biological-genetic vulnerability to schizophrenia? Schizophrenia Research, 54(1-2), 39-45. https://doi.org/10.1016/S0920-9964(01)00350-4

Walker, E., Lewis, N., Loewy, R., \& Palyo, S. (1999). Motor dysfunction and risk for schizophrenia. Development and Psychopathology, 11(3), 509-523.

Wang, Y., Wang, L., Xu, Q., Liu, D., Chen, L., Troje, N. F., He, S., \& Jiang, Y. (2018). Heritable aspects of biological motion perception and its covariation with autistic traits. Proceedings of the National Academy of Sciences of the United States of America. https://doi.org/10.1073/pnas.1714655115

Waters, F., Woodward, T., Allen, P., Aleman, A., \& Sommer, I. (2012). Self-recognition deficits in schizophrenia patients with auditory hallucinations: A meta-analysis of the literature. Schizophrenia Bulletin, 38(4), 741-750. https://doi.org/10.1093/schbul/sbq144

Wei, G., \& Luo, J. (2010). Sport expert's motor imagery: Functional imaging of professional motor skills and simple motor skills. Brain Research, 1341, 52-62. https://doi.org/10.1016/j.brainres.2009.08.014

Wohlschläger, A., Gattis, M., \& Bekkering, H. (2003). Action generation and action perception in imitation: An instance of the ideomotor principle. Philosophical Transactions of the Royal Society B: Biological Sciences, 358(1431), 501-515. https://doi.org/10.1098/rstb.2002.1257

Wolpert, D. M., \& Miall, R. C. (1996). Forward Models for Physiological Motor Control. Neural Networks.

Woodbury-Smith, M. R., Robinson, J., Wheelwright, S., \& Baron-Cohen, S. (2005). Screening adults for Asperger Syndrome using the AQ: A preliminary study of its diagnostic validity in clinical practice. Journal of Autism and Developmental Disorders. https://doi.org/10.1007/s10803-005$3300-7$ 
Running head: SELF-RECOGNITION OF ACTIONS

907 Wuthrich, V. M., \& Bates, T. C. (2006). Confirmatory factor analysis of the three-factor structure of the 908 Schizotypal Personality Questionnaire and Chapman schizotypy scales. Journal of Personality

909 Assessment, 87(3), 292-304. https://doi.org/10.1207/s15327752jpa8703_10

910 Zimmer, H. D., \& Engelkamp, J. (1996). Routes to Actions and their Efficacy for Remembering. 911 Memory, 4(1), 59-78. https://doi.org/10.1080/741940663 